\title{
Identification of Deleterious Mutations in Myostatin Gene of Rohu Carp (Labeo rohita) Using Modeling and Molecular Dynamic Simulation Approaches
}

\author{
Kiran Dashrath Rasal, Vemulawada Chakrapani, Swagat Kumar Patra, \\ Shibani D. Mohapatra, Swapnarani Nayak, Sasmita Jena, Jitendra Kumar Sundaray, \\ Pallipuram Jayasankar, and Hirak Kumar Barman
}

Fish Genetics and Biotechnology Division, Central Institute of Freshwater Aquaculture (ICAR), Bhubaneswar, Odisha 751 002, India

Correspondence should be addressed to Hirak Kumar Barman; hkbarman68@hotmail.com

Received 26 August 2015; Revised 13 January 2016; Accepted 27 January 2016

Academic Editor: Jian-Liang Li

Copyright (c) 2016 Kiran Dashrath Rasal et al. This is an open access article distributed under the Creative Commons Attribution License, which permits unrestricted use, distribution, and reproduction in any medium, provided the original work is properly cited.

\begin{abstract}
The myostatin (MSTN) is a known negative growth regulator of skeletal muscle. The mutated myostatin showed a doublemuscular phenotype having a positive significance for the farmed animals. Consequently, adequate information is not available in the teleosts, including farmed rohu carp, Labeo rohita. In the absence of experimental evidence, computational algorithms were utilized in predicting the impact of point mutation of rohu myostatin, especially its structural and functional relationships. The four mutations were generated at different positions (p.D76A, p.Q204P, p.C312Y, and p.D313A) of MSTN protein of rohu. The impacts of each mutant were analyzed using SIFT, I-Mutant 2.0, PANTHER, and PROVEAN, wherein two substitutions (p.D76A and p.Q204P) were predicted as deleterious. The comparative structural analysis of each mutant protein with the native was explored using 3D modeling as well as molecular-dynamic simulation techniques. The simulation showed altered dynamic behaviors concerning RMSD and RMSF, for either p.D76A or p.Q204P substitution, when compared with the native counterpart. Interestingly, incorporated two mutations imposed a significant negative impact on protein structure and stability. The present study provided the first-hand information in identifying possible amino acids, where mutations could be incorporated into MSTN gene of rohu carp including other carps for undertaking further in vivo studies.
\end{abstract}

\section{Introduction}

The myostatin, belonging to the member of the Transforming Growth Factor- $\beta$ (TGF $\beta$ ) superfamily, was initially identified in mice by McPherron et al. [1]. It is a negative regulator of skeletal muscle growth in mammalian species [2-5]. It inhibits myoblast specification and differentiation via downregulating Pax3, Myf-5, and MyoD expressions in myoblasts [6]. The mice carrying a targeted dominant mutation in MSTN gene resulted in the increased skeletal muscle mass due to the combined effects of muscle hypertrophy and hyperplasia $[7,8]$. Mice lacking the biologically active region of the MSTN C-terminal developed muscular hypertrophy in the chest and buttock [1]. Cattle (Bos taurus) is the first species in which a mutation in the MSTN gene showed the double-muscling phenotype. Subsequently, similar growth effects were reported in dogs, cattle, buffalo, porcine, and sheep [9-14]. In mammals, the desired effects were obtained due to mutation, positioned in either p.D76A, p.Q204A, p.C312Y, or p.C313Y. Therefore, the mutation mediated improvement of the skeletal muscle mass provided an avenue for improving growth related production traits in the farmed animals [15]. A transgenic mouse, containing a p.D76A mutation (within the leader peptide) in the MSTN gene, significantly increased muscle mass [16]. The experimentally incorporated mutations positioned at p.C313Y in cattle and p.D76A in mice as well as dog led to dramatic skeletal muscle growth.

The teleosts, MSTN gene, is well-conserved throughout evolution, where two distinct MSTN gene isoforms such as 
MSTNa and MSTNb were found in some fish species [1720]. In zebrafish (Danio rerio), the roles of MSTN gene concerning muscle development and growth were envisaged by inhibiting their function using its prodomain $[16,21,22]$. The MSTN gene characterization as well as knock-out and indel experiments in some fish species such that of yellow catfish [23], medaka [24], and rainbow trout [25] showed doublemuscling phenotype with hyperplasia and hypertrophy.

Thus, it would be of interest to identify each mutation in MSTN gene that disturbs its function, leading to improved muscle growth in commercially important aquaculture species. Commercial applications require in-depth analyses in the diversified teleosts. However, limited information is available whether the fish with mutated MSTN could display a similar phenotype or not. The Indian major carp, Labeo rohita (popularly known as rohu), is an economically important freshwater fish in India as well as other Asian countries [26-28]. The sequence data of rohu, catla (Catla catla), and zebrafish (Danio rerio) myostatin genes are available in the public domain (GenBank Acc. numbers HQ850573, HQ850575, and AY323521). However, its physiological functions in Indian major carps including rohu are lacking. In this study, we intended to assess the impact of p.D76A, p.Q204A, p.C312Y, and p.C313Y mutations in MSTN gene of rohu carp.

Direct experimental studies linked to incorporating mutation in a particular gene of interests have been laborious and time-consuming. The computational study can efficiently produce useful information to rationalize and guide in undertaking further experimental studies [28-30]. These point mutations affect gene expressions and their products by altering change in their functional activities [31]. The computational tools such as SIFT, PolyPhen, I-Mutant 2.0, PANTHER, and PROVEAN are more useful for the pin-pointing impact analysis of point mutation linking the gene function or gene products [29, 32-37]. Consequently, the combination of a different algorithm will improve the accuracy of results or predicted effects of particular mutation [38]. SNPs with amino acid (aa) substitutions can disturb protein folding and its stability, leading to altered protein function and proteinprotein interactions including its expression [39-42].

We have used several computational tools for determining the impact of the selected point mutation on MSTN gene. To get insight into the atomic-level changes and the dynamic behavior of the molecule on to the particular mutations, we have used molecular dynamic simulation approaches. We have identified the potential mutations, proposed modeled structure of the mutant proteins, and compared them with the native protein. The present in silico study is helpful for categorizing precise mutation in MSTN gene for the purpose of selecting a definite gene targeting site.

\section{Materials and Methods}

2.1. Retrieval of Data. The MSTN protein sequence of rohu, Labeo rohita, was retrieved from Uniprot with ID C9DE96_LABRO (http://www.uniprot.org/uniprot/C9DE96). SMART (a Simple Modular Architecture Research Tool) was used for identification and annotation including architectures of the mobile domain in the MSTN (http://smart.embl-heidelberg.de/).

2.2. Prediction of SNP Effects in MSTN Protein Using SequenceBased Tools. For elucidation of the impact of SNPs in MSTN protein of rohu, several in silico tools comprising of SIFT, PANTHER, PROVEAN, and I-Mutant 2.0 were utilized. The SIFT (Sorting Intolerant from Tolerant) algorithm is useful for determining the impact of single amino acid substitution in the resultant protein (http://sift.jcvi.org/). The tolerance index, that is, score of SIFT, in the tune of $\leq 0.05$, determined the nsSNPs (nonsynonymous SNPs) on resultant protein [43]. The higher the tolerance index, the less likely the impact on AASs. Subsequently, PANTHER (Protein Analysis through Evolutionary Relationships) was used so as to validate the predicted output from SIFT analysis. The PANTHER cSNP tool that estimates the likelihood of a particular nonsynonymous (amino acid changing) coding SNP to cause a functional impact on the protein (http://www.pantherdb.org/tools/csnp). It calculates the subPSEC score based on alignments of evolutionarily related proteins [44]. The score more than -3 is predicted as a less deleterious while less than -3 is predicted as deleterious. We have used this tool for detecting the impact of AASs at selected positions in the MSTN protein.

Further, PROVEAN and I-Mutant 2.0 algorithm were used for assessing the impacts of SNPs in MSTN protein of rohu. PROVEAN (Protein Variation Effect Analyzer) is a tool which predicts impact of an amino acid substitution or indel on the biological functions of a particular protein (http://provean.jcvi.org/index.php). This algorithm allows for the best-balanced separation between the deleterious and neutral AASs, based on a threshold value. A query sequence was provided in FASTA format and score for prediction was based on default threshold score -2.5 . The score $<-2.5$ indicates that variant is deleterious and $>-2.5$ is considered as a neutral [45]. I-Mutant 2.0 is a Support Vector Machine- (SVM-) based web server for the automatic prediction of protein stability changes upon single-site mutations (http://folding.biofold.org/i-mutant/i-mutant2.0.html). The predicted free energy change (DDG) is based on the differential unfolding Gibbs free energy change ( $\mathrm{kcal} / \mathrm{Mol}$ ) between mutant and native proteins [33].

2.3. MSTN Protein Structure Prediction. We have searched homologous 3D structure for MSTN protein of rohu in the RCSB PDB data bank. Due to the absence of homologous 3D structure, we have used I-TASSER (Iterative Threading ASSEmbly Refinement) server, which is an online platform for predicting protein structure and function (http://zhanglab.ccmb.med.umich.edu/I-TASSER/). It is based on multiple threading alignments and iterative structural assembly simulations. The prediction of the accuracy of the model depends upon a confidence score (C-score) determined by the quality of the threading alignments and structural assembly refinement simulations. In this server, we have submitted query sequence (FASTA format) of MSTN protein for obtaining 3D model. 
The best 3D model was selected according to rank based on C-score, TM-score, and RMSD value. The 3D structure of MSTN was visualized by PyMOL (http://www.pymol.org/) and UCSF-CHIMERA (https://www.cgl.ucsf.edu/chimera/). The PyMoL is an open source of molecular visualization tools, for interactive visualization and analysis of molecular structures. The superimposition of both MSTN native and mutant models was carried out by using UCSF-CHIMERA (https://www.cgl.ucsf.edu/chimera/). The SwissPDBViewer was used for analyzing RMS deviation by superimposing both native and mutant structures (http://spdbv.vital-it.ch/).

The structural evaluation and stereochemical quality assessments for both native and mutant models were carried out by using the SAVES (Structural Analysis and Verification Server), which is an integrated server (http://nihserver.mbi .ucla.edu/SAVES/). The ProSA (Protein Structure Analysis) web server (https://prosa.services.came.sbg.ac.at/prosa.php) was used for refinement and validation of protein structures [46]. The ProSA was used for checking model structural quality with potential errors, where program shows a plot of its residue energies and $Z$-scores to determine the overall quality of the model. The protein folding rate was calculated using PRORATE (prediction of protein folding rates) server (http://sunflower.kuicr.kyoto-u.ac.jp/ sjn/folding/).

\subsection{Investigation of SNP Effects in MSTN 3D Protein by} Structure-Based Tools. The mutant model was generated using PyMoL tool. The $\mathrm{H}$-bonding patterns were studied using the PyMoL visualization tool. Both native and mutant 3D structures of MSTN were used for molecular dynamic (MD) simulations.

The MD simulation was performed using the program of GROMACS 4.6.5 package (Groingen Machine for Chemical Simulations) running on Ubuntu Linux platform 14.04. Respective mutant and native models were solvated independently within a dodecahedron box with SPC216 (single point charge 216) water molecules at a marginal radius of $10 \AA$. The energy minimization was carried out for 5000 iterations/steps with a tolerance of $100 \mathrm{~kJ} / \mathrm{mol}$ by steepest descent algorithm (implementing OPLS force field). Emtol convergence criterion was set up to $1000 \mathrm{kcal} / \mathrm{Mol}$. The coulomb interactions were truncated at $1.0 \mathrm{~nm}$. The ionization states of the residues were set to a $\mathrm{pH}$ 7.0. Finally, MD simulation for 50000 steps (10 ns) was carried out. The resulted trajectory files were analyzed by using gromacs packages so as to differentiate RMSD and RMSF values between native and mutant structures of MSTN. The graphs were constructed using XMGrace on Linux platform itself.

\section{Results}

3.1. Mutation at Either p.D76A or p.Q204P of Rohu MSTN Protein Is Deleterious. Several computational tools were applied to investigate the structural and functional impacts of targeted single nucleotide polymorphisms (SNPs) present in the coding region of the MSTN gene of rohu (L. rohita). The dataset of possible nsSNPs was selected based upon previously reported observations in the mammalian system.
In this study, we have used both sequence-based (SIFT, PANTHER, PROVEAN, and I-Mutant) and structure-based (I-TASSER) algorithms. We have generated mutations by p.D76A, p.Q204P, p.C312Y, and p.D313A amino acid (aa) substitutions in the rohu MSTN protein using the PyMoL tool (Table 1). The most of the algorithms are based upon evolutionary principle. Subsequently, we have analyzed the effect of each mutation using a series of computational tools. Initially, SIFT, PANTHER, PROVEAN, and I-Mutant 2.0 analyses predicted p.D76A and p.Q204P substitutions as the most deleterious with score 0.02 and 0.04 , respectively (SIFT score $\leq$ 0.05). Contrary to this, p.C312Y and p.D313A mutations generated quite variable results, when analyzed with different algorithms of SIFT and PANTHER tools. The resultant significant differential outputs with SIFT and PANTHER algorithms could be due to consideration of different protein sequence alignments used to characterize the variants; the same was also documented earlier [47]. The functional impacts of aa substitutions (AAAs) were further validated by providing FASTA sequence of the protein as a query along with a change in residue(s) to PROVEAN, which is capable of classifying impacts of AASs or indels on functional protein [45]. Except p.D313A, the remaining three substitutions were classified as deleterious. To improve prediction accuracy, I-Mutant 2.0 algorithm was applied. This IMutant 2.0 also categorized p.C312Y with increased stability, while others were classified as deleterious substitutions.

\subsection{Structure-Based Analyses Also Supported the Deleterious} Nature of p.D76A and p.Q204A Substitutions. The structural information could play a vital role in unraveling the molecular mechanism as well as being helpful for predicting the effects of nsSNPs on overall protein structure [48]. The modeling of rohu MSTN protein was performed by I-TASSER, which is a structure-based automated prediction algorithm. Ten templates were considered for modeling MSTN protein. The best $3 \mathrm{D}$ structure with a high confidence score (C-score) was selected and used for further investigations. The top model, bearing estimated C-score of 0.46 , TM-score of $0.65 \pm$ 0.13 , and an RMSD value of $7.7 \pm 4.3 \AA$, was finally selected (Figure 1).

The differential Ramachandran plot is depicted in Figure 2. In the plot, native protein of phi/psi angles for $73.1 \%$ residues was in the additional allowed regions with $18.3 \%$ in the most favored regions, while being $8.6 \%$ in disallowed regions. The comparative analysis between native and mutant structure was carried out, which revealed that $72.8 \%, 18.3 \%$, and $8.9 \%$ residues lie in the most favored regions, additionally allowed regions, and disallowed regions, respectively. These results indicated that incorporated mutation enforced shifting of residues towards the disallowed region from the most favored regions in the case of p.Q204A mutation. No change was observed in p.D76A and native form of MSTN protein. The lowered ERRAT score for mutant in the tune of 78.797 as compared to native structure (83.476) also signified about the overall deteriorating quality of mutant MSTN (Figure 3). The ProSA analysis showed slightly deviated $Z$ score value in mutant protein (p.D76A and p.Q204A) as compared to native one. The score for native was -4.41 , while 


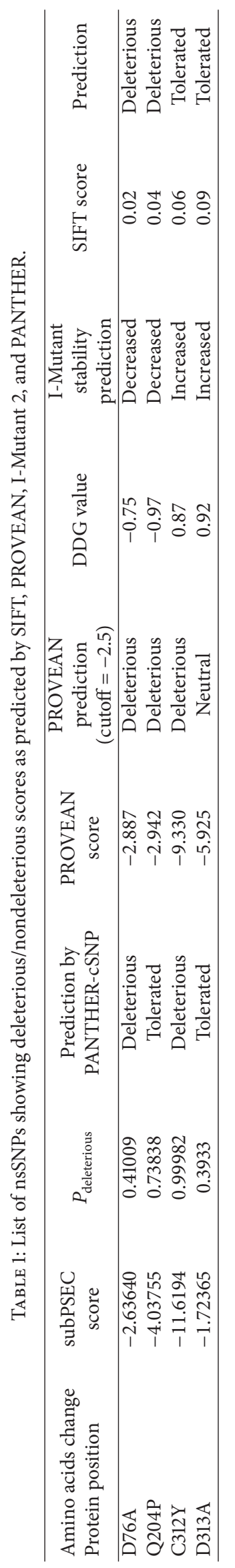



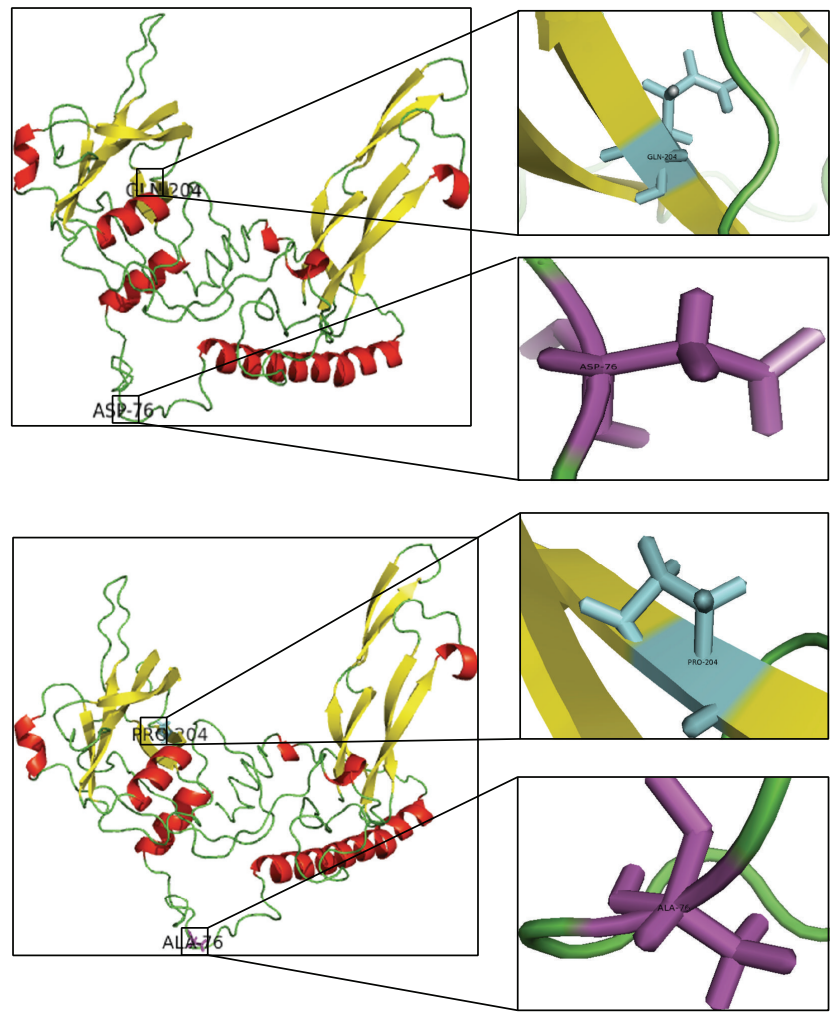

FIGURE 1: Predicted MSTN models by I-TASSER (both native and mutant) with mutated native and mutated residues.

mutants p.D76A and p.Q204A were -4.36 and 4.43, respectively. Together, these results provided the clue regarding possible conformational alterations for both mutant proteins (p.D76A and p.Q204A). The analysis of protein folding rate also specified that p.Q204A mutant and native possessed -18.38321 , whereas folding rate varied with mutant p.D76A $(-18.410992)$. It can be inferred from the structural analysis that the two deleterious mutations (p.D76A and p.Q204A) had fetched a severe change in the MSTN protein, and it is likely to disturb the protein function.

3.3. Analysis of Simulation at the Atomic Level Predicted the Deviated Functioning of Mutated (p.D76A or p.Q204A) $M S T N$. In order to gain atomic-level insights as well as understanding of dynamic behavioral changes in mutant proteins, we performed MD simulations for both the native and mutant MSTNs. The MD simulation has been known to support the predictions given by sequence-based in silico tools. For this purpose, the RMSD of the protein backbone as a mean for functional time was examined. The RMSD for all protein- $\mathrm{H}$ atoms from the initial structure was reexamined to study the convergence of the protein system (Figure 4(a)). The mutant RMSD trajectory detected an abrupt rise of RMSD value starting from 5 ps onwards. The RMSD graph showed higher deviation in MSTN mutant (p.D76A and p.Q204P) structures as compared to the native counterpart (Figure 4(a)). To assess the effect of mutation on the MSTN structure, we also computed the RMSF values. The graphs show higher RMS fluctuations in MSTN mutant (both p.D76A and p.Q204P) structures as compared to the native structure (Figure 4(b)). Superimposition of native and mutant structures detected the RMS deviations in the tune of $0.049 \AA, 0.029 \AA, 0.030 \AA$, and $0.024 \AA$, respectively, for p.D76A, p.Q204P, p.C312Y, and p.D313A. The varied potential energies between native and both forms of mutants were also documented. The p.D76A mutant possessed $-1.1378342 e+$ $06 \mathrm{~kJ} / \mathrm{Mol}$ and p.Q204P mutant contained $-1.1424335 e+$ $06 \mathrm{~kJ} / \mathrm{Mol}$ (p.Q204P), whereas it possessed comparatively lesser potential energy in the tune of $-1.170186 e+06 \mathrm{~kJ} / \mathrm{Mol}$ for native counterpart. Further, the calculated total energies after $\mathrm{MD}$ simulated production run were $-7.86233 e+$ $05 \mathrm{~kJ} / \mathrm{Mol},-7.89900 e+05 \mathrm{~kJ} / \mathrm{Mol}$, and $-8.4884 e+05 \mathrm{~kJ} / \mathrm{Mol}$, respectively, for p.D76A mutant, p.Q204P mutant, and the native structures. Thus, the differentially heightened energy requirements for both the mutant structures as compared to native one were suggestive of affecting (disturbed) the proper functioning of mutant MSTNs.

\section{Discussion}

Several evidences are available that the inhibition of MSTN gene led to dramatic growth rate/double-muscling phenotype in mammals. Alongside, attempts have been made to attenuate MSTN activities in fish muscle using mutagenesis approach. But, those approaches in fishes led to conflicting outcomes, possibly due to duplicated gene events or faulty selection of mutation sites. Also, the MSTN protein is extremely well-conserved between fish and other vertebrates. 


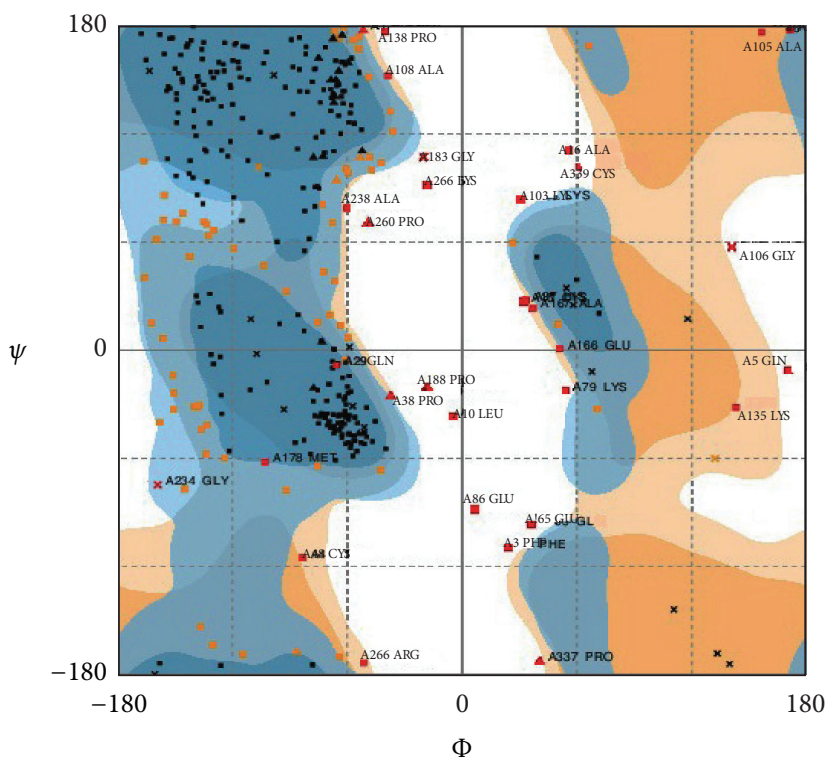

(a)

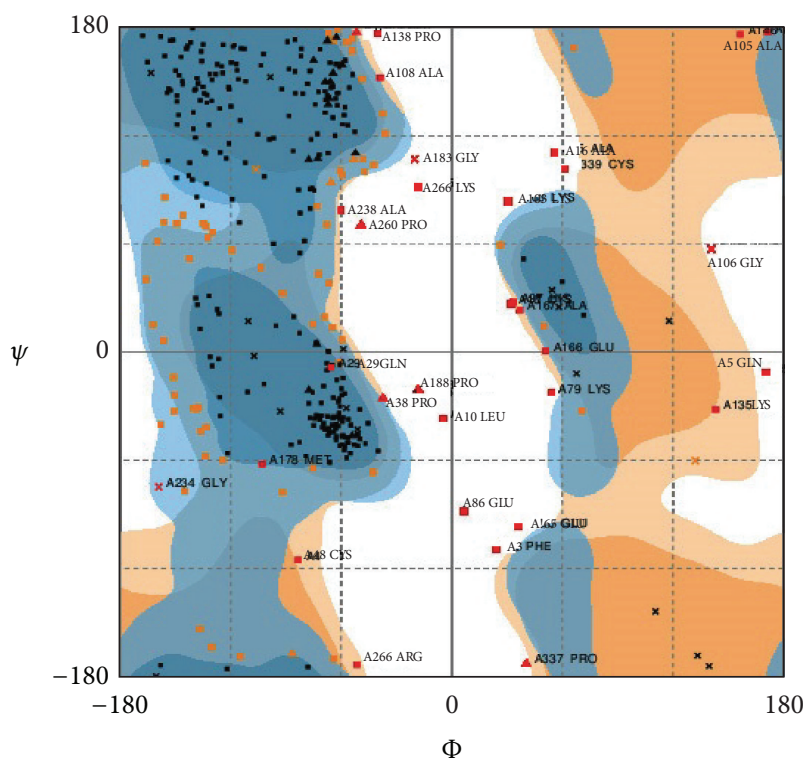

(b)

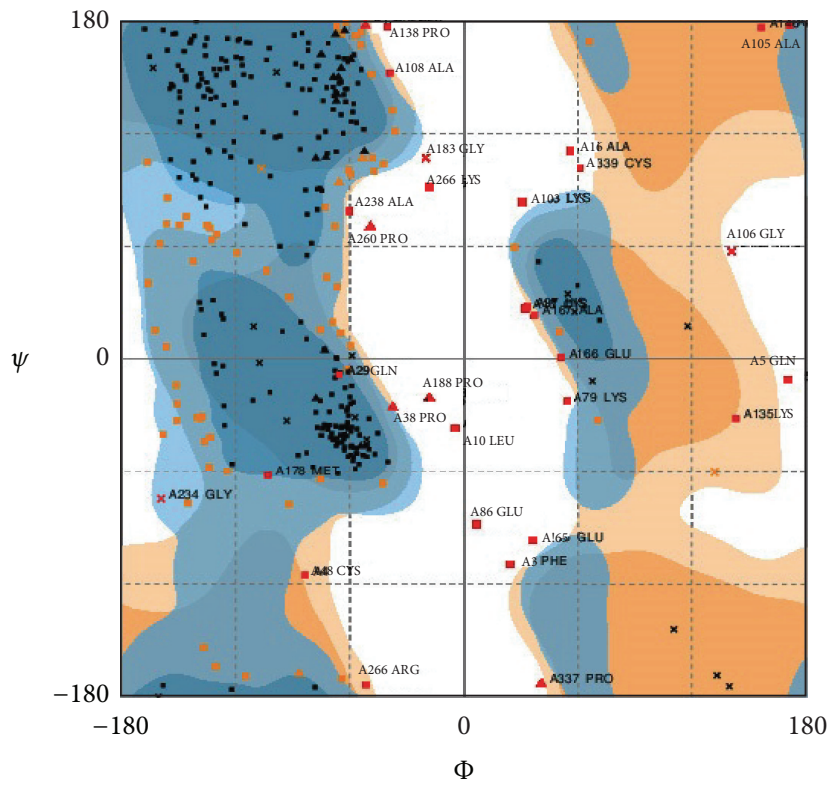

- $\Delta$ General/pre-pro/proline favored

$\times$ Glycine favored

I $\mathrm{A}$ General/pre-pro/proline allowed

Glycine allowed

(c)

FIGURE 2: Ramachandran plot of MSTN mutant protein models ( $\mathrm{a}, \mathrm{b}$, and c). The most favored regions, additional allowed regions, generously allowed regions, and disallowed regions are indicated as dark blue, light blue, light yellow, and white, respectively.

Nearly $90 \%$ of the amino acids (aa) are identical between fish and mammalian MSTN sequences. The experimental evidence in zebrafish [22] and medaka [49] demonstrated the increased number of skeletal muscle fibers via inhibition of MSTN. Remarkably, inhibition of MSTN could not lead to elevated muscle weight in both cases. Therefore, extensive studies are essential so as to understand the roles being played by MSTN in farmed fishes. The impact analysis as a consequence of mutation at a precise location is very important in order to gain economic benefits from farmed fishes.

Computational biology has long been a guiding force for undertaking experimental investigations. In the present work, we have generated mutations by amino acid substitutions at four positions such as p.D76A or p.Q204P or p.C312Y or p.D313A. SIFT, PANTHER, PROVEAN, and IMutant 2.0 based analyses predicted p.D76A and p.Q204P 


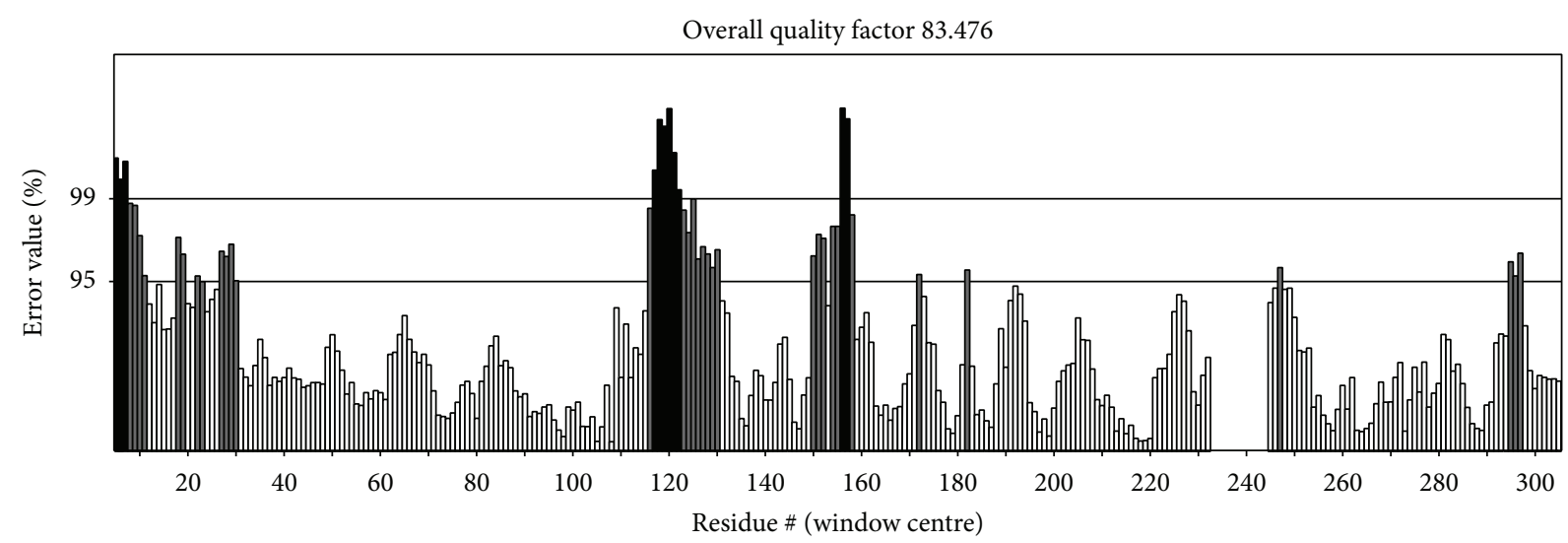

(a)

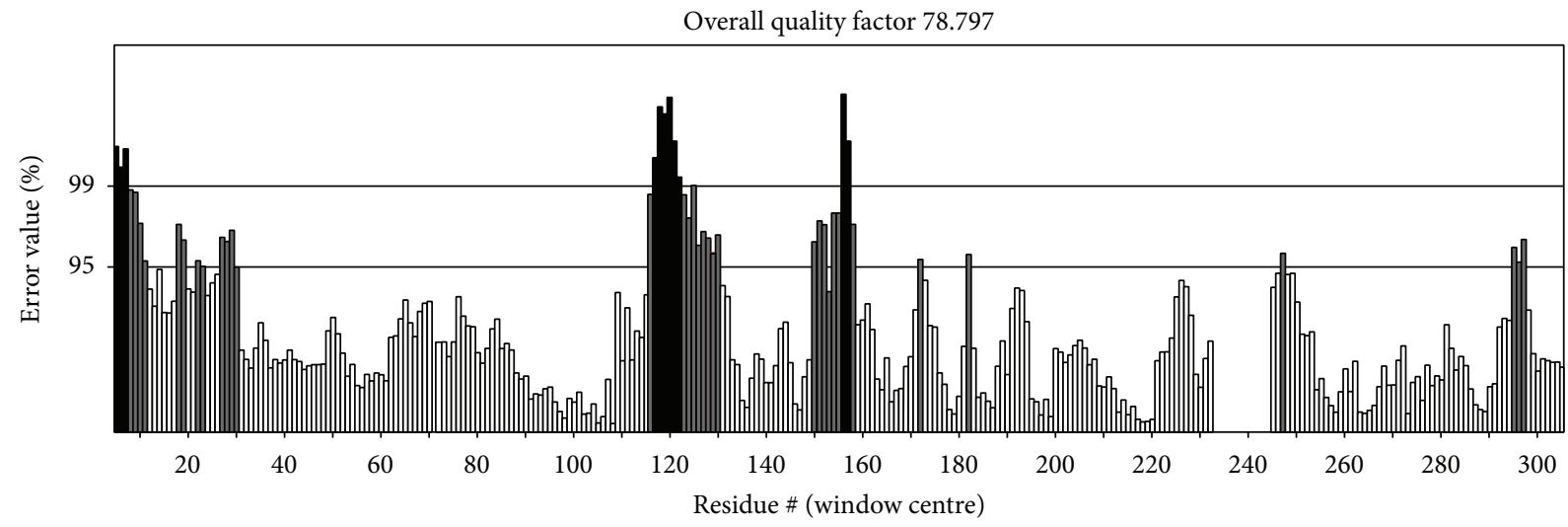

(b)

FIGURE 3: ERRAT plots for (a) MSTN native protein and (b) MSTN mutant protein model generated by SAVES. The plots for both proteins show overall quality factors 83.476 and 78.797 , respectively. The grey region indicates error region $95 \%$ and $99 \%$, white bars show region of lower error rate for protein folding, and black bars show misfolded region located distantly from active site. The below $95 \%$ value (i.e., rejection limit) indicates low resolution structure, while more than or $95 \%$ value shows high resolution structure.

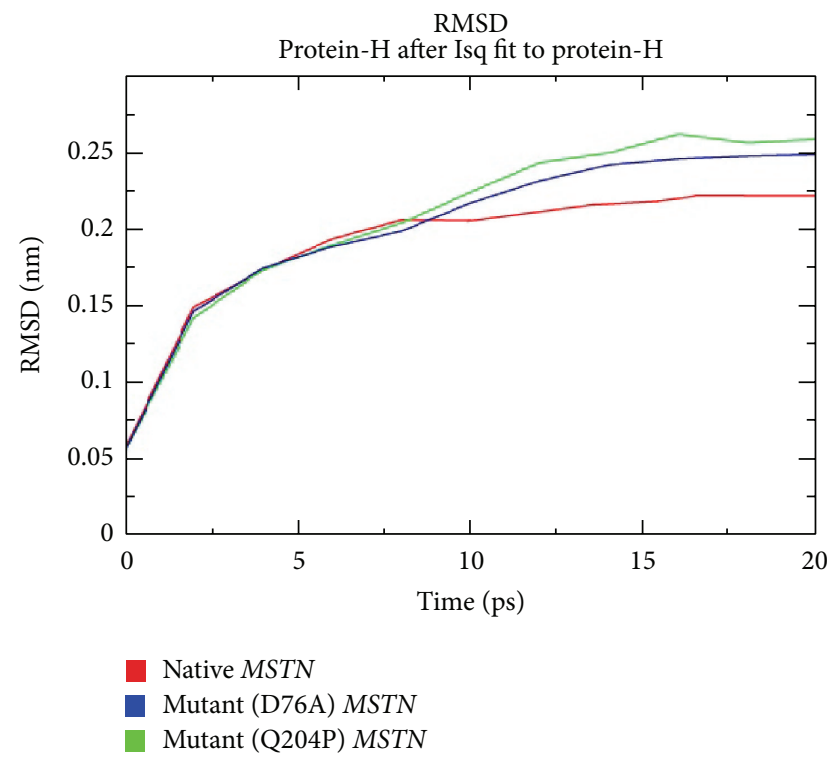

(a)

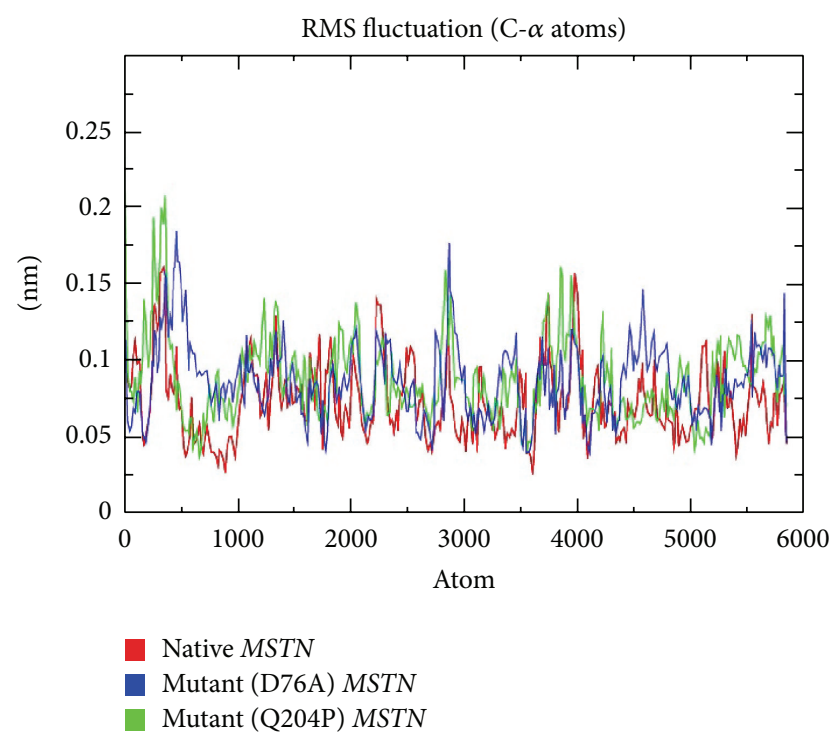

(b)

FIGURE 4: The RMSD and RMSF of both native and mutant MSTN structures of rohu carp (red color: native; blue: mutant MSTN (p.D76A); and green: mutant (p.Q204P). 
substitutions as the most deleterious. Those computational methods can predict (based on evolutionary principles) the effect of nsSNPs in the coding region of the genome [35, 5052]. The $3 \mathrm{D}$ structure of a protein has been very helpful in predicting the effects of nsSNPs on protein structure-function $[43,48]$. We have generated 3D structure of MSTN and validated it by using Ramachandran plot. Further, through MD simulation, we have compared RMSD values and total energy values between native and mutant structures. Based on estimated RMSD values, the computed energy provided the comparative information regarding structural stability and the deviation between the two (native and each mutant) structures. The deviated RMSD could have an impact on the functional activity and stability of protein [53]. The rise in RMSD values in the graph indicated that there was an enormous deviation between the native and mutant structures, pointing towards alterations in the structural and functional stabilities. We thus inferred that these nsSNPs could affect the stability of MSTN structure of rohu. Thus, it is likely that either p.D76A or p.Q204P substitution was deleterious in nature with regard to MSTN gene function. Our findings are partly in line with earlier experimental findings that a transgenic mouse, bearing p.D76A MSTN gene mutation, showed significantly improved muscle mass [16]. Also, mutations in the position p.Q204X, producing a premature stop codon in the N-terminal domain, resulted into double-muscling phenotype in Charolais and Limousine cattle breeds [54].

Thus, we could find that point mutation in the MSTN gene disturbs the protein structure and affects its function. Our findings also suggested that mutations in the N-terminal motif of TFGB chain could more effectively inhibit the MSTN gene activity. Our findings will provide a clue that improvement in muscular mass could be experimentally possible by incorporating p.D76A and/or p.Q204P mutations in rohu MSTN gene.

As the transcriptional regulation of MSTN genes is mediated by different myogenic factors, the previous works demonstrated that MSTN could able to negatively autoregulate its expression through a Smad7-dependent mechanism [55].

In recent times, gene targeting in predetermined position in the genome was made possible using technologies of zincfinger nucleases (ZFNs), transcription activator-like effector nucleases (TALENs), and CRISPR/Cas9 in wide ranges of cell lines [56-63]. The site-specific nick by nucleases can then be repaired by error-prone nonhomologous end joining (NHEJ) resulting in animals carrying deletions or insertions at the cut site. It is also possible to disrupt any gene of interests by enforcing homologous recombination (HR) mediated repair at a particular nick site. Thus, it is possible to generate model fishes by creating indels and/or gene disruptions in the myostatin gene. This in turn will lead not only to uncover physiological roles of the myostatin gene but also to improve skeletal muscle growth in Indian farmed carps.

\section{Conclusions}

Our computational study identified that p.D76A or p.Q204P amino acid substitutions (nsSNPs) are deleterious for the structural and functional aspects of the rohu (L. Rohita)
MSTN protein. We investigated the impact of the four variants on MSTN in the form of energy calculation and evolutionarily conserved residues. The SIFT, PANTHER, PROVEAN, and I-Mutant algorithms predicted decrease in protein stability. The MD simulation also confirmed that change in the dynamic behavior of mutant protein at the atomic level to the native counterpart. Based on the documented differences in total energy for native and mutant models, we speculated that the proper functioning of native MSTN protein would be disturbed.Our results suggested that these mutants in $\mathrm{TGFb}$ domain have a potential functional impact on MSTN genes in teleosts. The generated data from this study will be resourceful for undertaking future in vivo investigations so as to improve the growtgh rate of famed carps.

\section{Abbreviations \\ MSTN: Myostatin \\ SIFT: Sorting Intolerant from Tolerant \\ SNP: Single nucleotide polymorphism \\ RMSD: Root means square deviations \\ PDB: Protein data bank \\ RMSF: Root means square fluctuations \\ MD: Molecular dynamics.}

\section{Conflict of Interests}

The authors have no potential conflict of interests to disclose and no competing financial interests exist.

\section{Authors' Contribution}

Hirak Kumar Barman, Pallipuram Jayasankar, and Jitendra Kumar Sundaray conceived and designed experiments. Kiran Dashrath Rasal, Vemulawada Chakrapani, Swagat Kumar Patra, Shibani D. Mohapatra, Swapnarani Nayak, and Sasmita Jena performed the experiment and analyzed data. Kiran Dashrath Rasal, Vemulawada Chakrapani, Swagat Kumar Patra, and Hirak Kumar Barman wrote the paper.

\section{Acknowledgment}

The work was supported by the Indian Council of Agricultural Research, Ministry of Agriculture, Government of India.

\section{References}

[1] A. C. McPherron, A. M. Lawler, and S.-J. Lee, "Regulation of skeletal muscle mass in mice by a new TGF- $\beta$ superfamily member," Nature, vol. 387, no. 6628, pp. 83-90, 1997.

[2] A. Stinckens, M. Georges, and N. Buys, "Mutations in the Myostatin gene leading to hypermuscularity in mammals: indications for a similar mechanism in fish?" Animal Genetics, vol. 42, no. 3, pp. 229-234, 2011.

[3] A. M. Haidet, L. Rizo, C. Handy et al., "Long-term enhancement of skeletal muscle mass and strength by single gene administration of myostatin inhibitors," Proceedings of the National 
Academy of Sciences of the United States of America, vol. 105, no. 11, pp. 4318-4322, 2008.

[4] S.-J. Lee, "Regulation of muscle mass by myostatin," Annual Review of Cell and Developmental Biology, vol. 20, pp. 61-86, 2004.

[5] H. J. Xing, Z. Y. Wang, B. S. Zhong et al., "Effects of different dietary intake on mRNA levels of MSTN, IGF-I, and IGF-II in the keletal muscle of Dorper and Hu sheep hybrid $\mathrm{F}_{1}$ rams," Genetics and Molecular Research, vol. 13, no. 3, pp. 5258-5268, 2014.

[6] B. Langley, M. Thomas, A. Bishop, M. Sharma, S. Gilmour, and R. Kambadur, "Myostatin inhibits myoblast differentiation by down-regulating MyoD expression," The Journal of Biological Chemistry, vol. 277, no. 51, pp. 49831-49840, 2002.

[7] G. Szabó, G. Dallmann, G. Müller, L. Patthy, M. Soller, and L. Varga, "A deletion in the myostatin gene causes the compact (Cmpt) hypermuscular mutation in mice," Mammalian Genome, vol. 9, no. 8, pp. 671-672, 1998.

[8] X. Zhu, M. Hadhazy, M. Wehling, J. G. Tidball, and E. M. McNally, "Dominant negative myostatin produces hypertrophy without hyperplasia in muscle," FEBS Letters, vol. 474, no. 1, pp. 71-75, 2000.

[9] A. Stinckens, T. Luyten, J. Bijttebier et al., "Characterization of the complete porcine MSTN gene and expression levels in pig breeds differing in muscularity," Animal Genetics, vol. 39, no. 6, pp. 586-596, 2008.

[10] D. S. Mosher, P. Quignon, C. D. Bustamante et al., "A mutation in the myostatin gene increases muscle mass and enhances racing performance in heterozygote dogs," PLoS Genetics, vol. 3, no. 5, article e79, 2007.

[11] I. A. Boman, G. Klemetsdal, O. Nafstad, T. Blichfeldt, and D. I. Våge, "Impact of two myostatin (MSTN) mutations on weight gain and lamb carcass classification in Norwegian White Sheep (Ovis aries)," Genetics Selection Evolution, vol. 42, article 4, 2010.

[12] Y. Chen, J. Ye, L. Cao, Y. Zhang, W. Xia, and D. Zhu, "Myostatin regulates glucose metabolism via the AMP-activated protein kinase pathway in skeletal muscle cells," The International Journal of Biochemistry \& Cell Biology, vol. 42, no. 12, pp. 20722081, 2010.

[13] S. Hu, W. Ni, W. Sai et al., "Knockdown of myostatin expression by RNAi enhances muscle growth in transgenic sheep," PLoS ONE, vol. 8, no. 3, Article ID e58521, 2013.

[14] D.-S. Han, C.-S. Yu, C.-K. Chiang et al., "Serum myostatin is reduced in individuals with metabolic syndrome," PLOS ONE, vol. 9, no. 9, Article ID e108230, 2014.

[15] C. Rehfeldt, M. F. W. Te Pas, K. Wimmers et al., "Advances in research on the prenatal development of skeletal muscle in animals in relation to the quality of muscle-based food. I. Regulation of myogenesis and environmental impact," Animal, vol. 5, no. 5, pp. 703-717, 2011.

[16] S.-J. Lee, "Genetic analysis of the role of proteolysis in the activation of latent myostatin," PLOS ONE, vol. 3, no. 2, Article ID e1628, 2008.

[17] P. R. Biga, S. B. Roberts, D. B. Iliev et al., "The isolation, characterization, and expression of a novel GDF11 gene and a second myostatin form in zebrafish, Danio rerio," Comparative Biochemistry and Physiology. Part B: Biochemistry and Molecular Biology, vol. 141, no. 2, pp. 218-230, 2005.

[18] T. Kerr, E. H. Roalson, and B. D. Rodgers, "Phylogenetic analysis of the myostatin gene sub-family and the differential expression of a novel member in zebrafish," Evolution \& Development, vol. 7, no. 5, pp. 390-400, 2005.
[19] S. B. Roberts and F. W. Goetz, "Differential skeletal muscle expression of myostatin across teleost species, and the isolation of multiple myostatin isoforms," FEBS Letters, vol. 491, no. 3, pp. 212-216, 2001.

[20] B. Funkenstein, V. Balas, Y. Rebhan, and A. Pliatner, "Characterization and functional analysis of the 5/ flanking region of Sparus aurata myostatin-1 gene," Comparative Biochemistry and Physiology - A Molecular \& Integrative Physiology, vol. 153, no. 1, pp. 55-62, 2009.

[21] B. M. Meyer, J. M. Froehlich, N. J. Galt, and P. R. Biga, "Inbred strains of zebrafish exhibit variation in growth performance and myostatin expression following fasting," Comparative Biochemistry and Physiology Part A: Molecular \& Integrative Physiology, vol. 164, no. 1, pp. 1-9, 2013.

[22] C. Xu, G. Wu, Y. Zohar, and S.-J. Du, "Analysis of myostatin gene structure, expression and function in zebrafish," The Journal of Experimental Biology, vol. 206, no. 22, pp. 4067-4079, 2003.

[23] Z. Dong, J. Ge, K. Li et al., "Heritable targeted inactivation of myostatin gene in yellow catfish (Pelteobagrus fulvidraco) using engineered zinc finger nucleases," PLoS ONE, vol. 6, no. 12, Article ID e28897, 2011.

[24] S.-I. Chisada, H. Okamoto, Y. Taniguchi et al., "Myostatindeficient medaka exhibit a double-muscling phenotype with hyperplasia and hypertrophy, which occur sequentially during post-hatch development," Developmental Biology, vol. 359, no. 1, pp. 82-94, 2011.

[25] M. P. Phelps, I. M. Jaffe, and T. M. Bradley, "Muscle growth in teleost fish is regulated by factors utilizing the activin II B receptor," The Journal of Experimental Biology, vol. 216, no. 19, pp. 3742-3750, 2013.

[26] H. K. Barman, V. Das, R. Mohanta, C. Mohapatra, R. P. Panda, and P. Jayasankar, "Expression analysis of $\beta$-actin promoter of rohu (Labeo rohita) by direct injection into muscle," Current Science, vol. 99, no. 8, pp. 1030-1032, 2010.

[27] H. K. Barman, S. K. Patra, V. Das et al., "Identification and characterization of differentially expressed transcripts in the gills of freshwater prawn (Macrobrachium rosenbergii) under salt stress," The Scientific World Journal, vol. 2012, Article ID 149361, 11 pages, 2012.

[28] C. Mohapatra, H. K. Barman, R. P. Panda et al., "Cloning of cDNA and prediction of peptide structure of Plzf expressed in the spermatogonial cells of Labeo rohita," Marine Genomics, vol. 3, no. 3-4, pp. 157-163, 2010.

[29] C. G. P. Doss and B. Rajith, "Computational refinement of functional single nucleotide polymorphisms associated with ATM gene," PLoS ONE, vol. 7, no. 4, Article ID e34573, 2012.

[30] K. D. Rasal, V. Chakrapani, S. K. Patra et al., "Identification and prediction of the consequences of nonsynonymous SNPs in glyceraldehyde 3-phosphate dehydrogenase (GAPDH) gene of zebrafish Danio rerio," Turkish Journal of Biology, vol. 40, pp. 43-54, 2016.

[31] P. D. Thomas and A. Kejariwal, "Coding single-nucleotide polymorphisms associated with complex vs. Mendelian disease: evolutionary evidence for differences in molecular effects," Proceedings of the National Academy of Sciences of the United States of America, vol. 101, no. 43, pp. 15398-15403, 2004.

[32] M. M. Johnson, J. Houck, and C. Chen, "Screening for deleterious nonsynonymous single-nucleotide polymorphisms in genes involved in steroid hormone metabolism and response," Cancer Epidemiology Biomarkers and Prevention, vol. 14, no. 5, pp. 1326-1329, 2005. 
[33] E. Capriotti, P. Fariselli, and R. Casadio, "I-Mutant2.0: predicting stability changes upon mutation from the protein sequence or structure," Nucleic Acids Research, vol. 33, no. 2, pp. W306W310, 2005.

[34] C. George Priya Doss, R. Rajasekaran, C. Sudandiradoss, K. Ramanathan, R. Purohit, and R. Sethumadhavan, "A novel computational and structural analysis of nsSNPs in CFTR gene," Genomic Medicine, vol. 2, no. 1-2, pp. 23-32, 2008.

[35] M. R. M. Hussain, N. A. Shaik, J. Y. Al-Aama et al., "In silico analysis of Single Nucleotide Polymorphisms (SNPs) in human BRAF gene," Gene, vol. 508, no. 2, pp. 188-196, 2012.

[36] H. S. H. A. Mohamoud, M. R. Hussain, A. A. El-Harouni et al., "First comprehensive in silico analysis of the functional and structural consequences of SNPs in human GalNAc-T1 gene," Computational and Mathematical Methods in Medicine, vol. 2014, Article ID 904052, 15 pages, 2014.

[37] K. D. Rasal, T. M. Shah, M. Vaidya, S. J. Jakhesara, and C. G. Joshi, "Analysis of consequences of non-synonymous SNP in feed conversion ratio associated TGF- $\beta$ receptor type 3 gene in chicken," Meta Gene, vol. 4, pp. 107-117, 2015.

[38] L. G. Martelotto, C. K. Ng, M. R. De Filippo et al., "Benchmarking mutation effect prediction algorithms using functionally validated cancer-related missense mutations," Genome Biology, vol. 15, article 484, 2014.

[39] R. De Cristofaro, A. Carotti, S. Akhavan et al., "The natural mutation by deletion of Lys 9 in the thrombin A-chain affects the pKa value of catalytic residues, the overall enzyme's stability and conformational transitions linked to $\mathrm{Na}^{+}$binding," The FEBS Journal, vol. 273, no. 1, pp. 159-169, 2006.

[40] M. Hardt and R. A. Laine, "Mutation of active site residues in the chitin-binding domain $\mathrm{ChBD}_{\mathrm{ChiAl}}$ from chitinase $\mathrm{Al}$ of Bacillus circulans alters substrate specificity: use of a green fluorescent protein binding assay," Archives of Biochemistry and Biophysics, vol. 426, no. 2, pp. 286-297, 2004.

[41] R. Jones, M. Ruas, F. Gregory et al., "A CDKN2A mutation in familial melanoma that abrogates binding of p16INK4a to CDK4 but not CDK6," Cancer Research, vol. 67, pp. 9134-9141, 2007.

[42] H. Ode, S. Matsuyama, M. Hata et al., "Computational characterization of structural role of the non-active site mutation M36I of human immunodeficiency virus type 1 protease," Journal of Molecular Biology, vol. 370, no. 3, pp. 598-607, 2007.

[43] P. C. Ng and S. Henikoff, "Predicting the effects of amino acid substitutions on protein function," Annual Review of Genomics and Human Genetics, vol. 7, pp. 61-80, 2006.

[44] P. D. Thomas, M. J. Campbell, A. Kejariwal et al., "PANTHER: a library of protein families and subfamilies indexed by function," Genome Research, vol. 13, no. 9, pp. 2129-2141, 2003.

[45] Y. Choi, G. E. Sims, S. Murphy, J. R. Miller, and A. P. Chan, "Predicting the functional effect of amino acid substitutions and indels," PLoS ONE, vol. 7, no. 10, Article ID e46688, 2012.

[46] M. Wiederstein and M. J. Sippl, "ProSA-web: interactive web service for the recognition of errors in three-dimensional structures of proteins," Nucleic Acids Research, vol. 35, no. 2, pp. W407-W410, 2007.

[47] S. A. De Alencar and J. C. D. Lopes, "A comprehensive in silico analysis of the functional and structural impact of SNPs in the IGF1R gene," Journal of Biomedicine and Biotechnology, vol. 2010, Article ID 715139, 8 pages, 2010.

[48] P. Kumar, S. Henikoff, and P. C. Ng, "Predicting the effects of coding non-synonymous variants on protein function using the
SIFT algorithm," Nature Protocols, vol. 4, no. 7, pp. 1073-1082, 2009.

[49] E. Sawatari, R. Seki, T. Adachi et al., "Overexpression of the dominant-negative form of myostatin results in doubling of muscle-fiber number in transgenic medaka (Oryzias latipes)," Comparative Biochemistry and Physiology. PartA: Molecular \& Integrative Physiology, vol. 155, no. 2, pp. 183-189, 2010.

[50] M. Alanazi, Z. Abduljaleel, W. Khan et al., "In silico analysis of single nucleotide polymorphism (snps) in human $\beta$-globin gene," PLoS ONE, vol. 6, no. 10, Article ID e25876, 2011.

[51] S. Khan and M. Vihinen, "Performance of protein stability predictors," Human Mutation, vol. 31, no. 6, pp. 675-684, 2010.

[52] B. Rajith and C. G. P. Doss, "Path to facilitate the prediction of functional amino acid substitutions in red blood cell disorders-a computational approach," PLoS ONE, vol. 6, no. 9, Article ID e24607, 2011.

[53] J.-H. Han, N. Kerrison, C. Chothia, and S. A. Teichmann, "Divergence of interdomain geometry in two-domain proteins," Structure, vol. 14, no. 5, pp. 935-945, 2006.

[54] R. Kambadur, M. Sharma, T. P. L. Smith, and J. J. Bass, "Mutations in myostatin (GDF8) in double-muscled Belgian Blue and Piedmontese cattle," Genome Research, vol. 7, no. 9, pp. 910-916, 1997.

[55] C. McFarlane, A. Vajjala, H. Arigela et al., "Negative autoregulation of myostatin expression is mediated by $S m a d 3$ and microRNA-27," PLoS ONE, vol. 9, no. 1, Article ID e87687, 2014.

[56] A. R. Bassett and J.-L. Liu, "CRISPR/Cas9 and genome editing in Drosophila," Journal of Genetics and Genomics, vol. 41, no. 1, pp. 7-19, 2014.

[57] T. Gaj, C. A. Gersbach, and C. F. Barbas III, "ZFN, TALEN, and CRISPR/Cas-based methods for genome engineering," Trends in Biotechnology, vol. 31, no. 7, pp. 397-405, 2013.

[58] D. Hockemeyer, H. Wang, S. Kiani et al., "Genetic engineering of human pluripotent cells using TALE nucleases," Nature Biotechnology, vol. 29, no. 8, pp. 731-734, 2011.

[59] W. Y. Hwang, Y. Fu, D. Reyon et al., "Efficient genome editing in zebrafish using a CRISPR-Cas system," Nature Biotechnology, vol. 31, no. 3, pp. 227-229, 2013.

[60] M. Kanatsu-Shinohara, M. Kato-Itoh, M. Ikawa et al., "Homologous recombination in rat germline stem cells," Biology of Reproduction, vol. 85, no. 1, pp. 208-217, 2011.

[61] A. Klug, "The discovery of zinc fingers and their development for practical applications in gene regulation and genome manipulation," Quarterly Reviews of Biophysics, vol. 43, no. 1, pp. 1-21, 2010.

[62] P. Mali, J. Aach, P. B. Stranges et al., "CAS9 transcriptional activators for target specificity screening and paired nickases for cooperative genome engineering," Nature Biotechnology, vol. 31, no. 9, pp. 833-838, 2013.

[63] N. E. Sanjana, L. Cong, Y. Zhou, M. M. Cunniff, G. Feng, and F. Zhang, "A transcription activator-like effector toolbox for genome engineering," Nature Protocols, vol. 7, no. 1, pp. 171-192, 2012. 

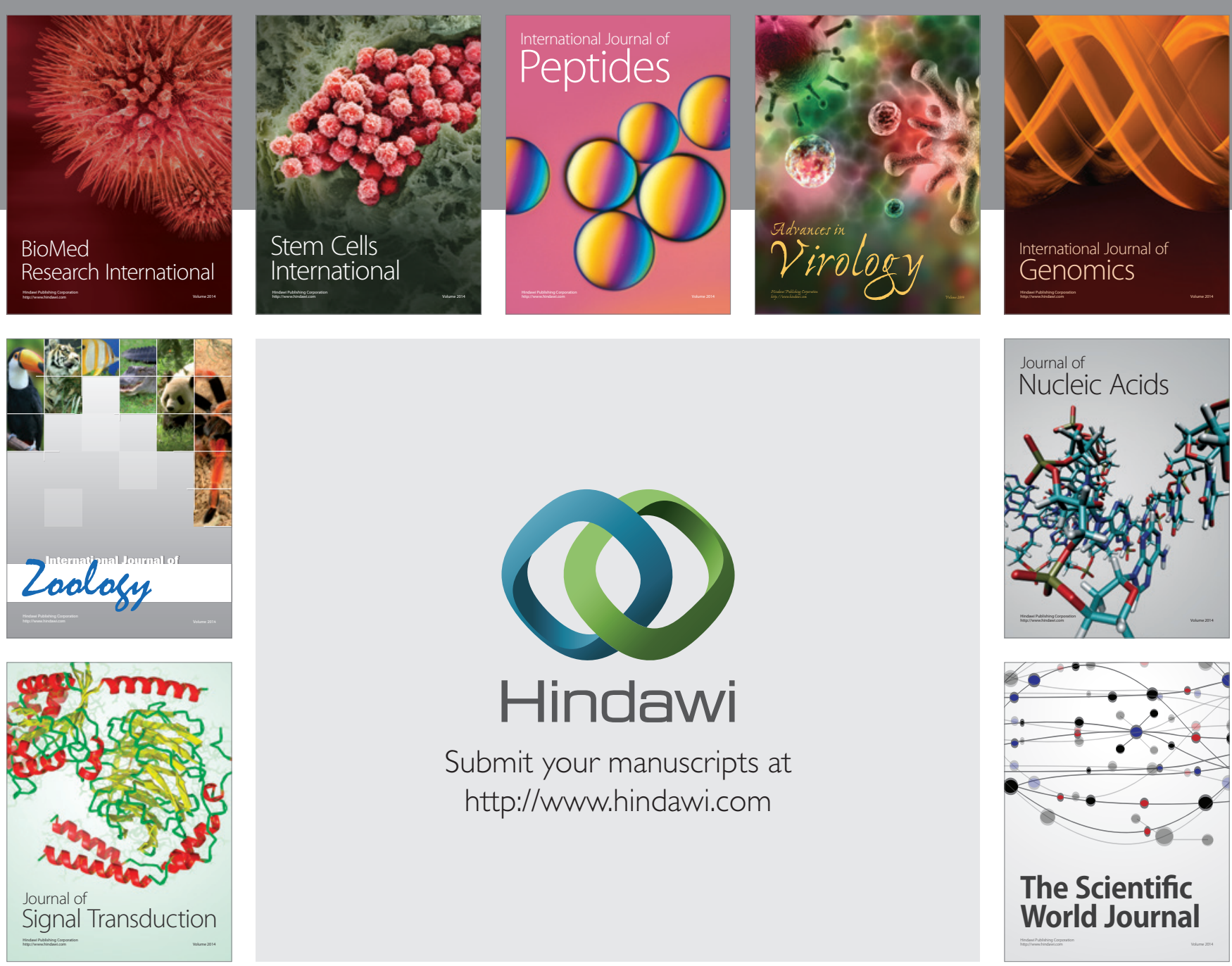

Submit your manuscripts at

http://www.hindawi.com
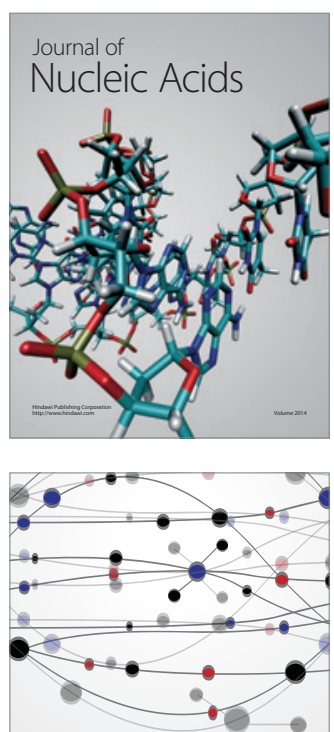

The Scientific World Journal
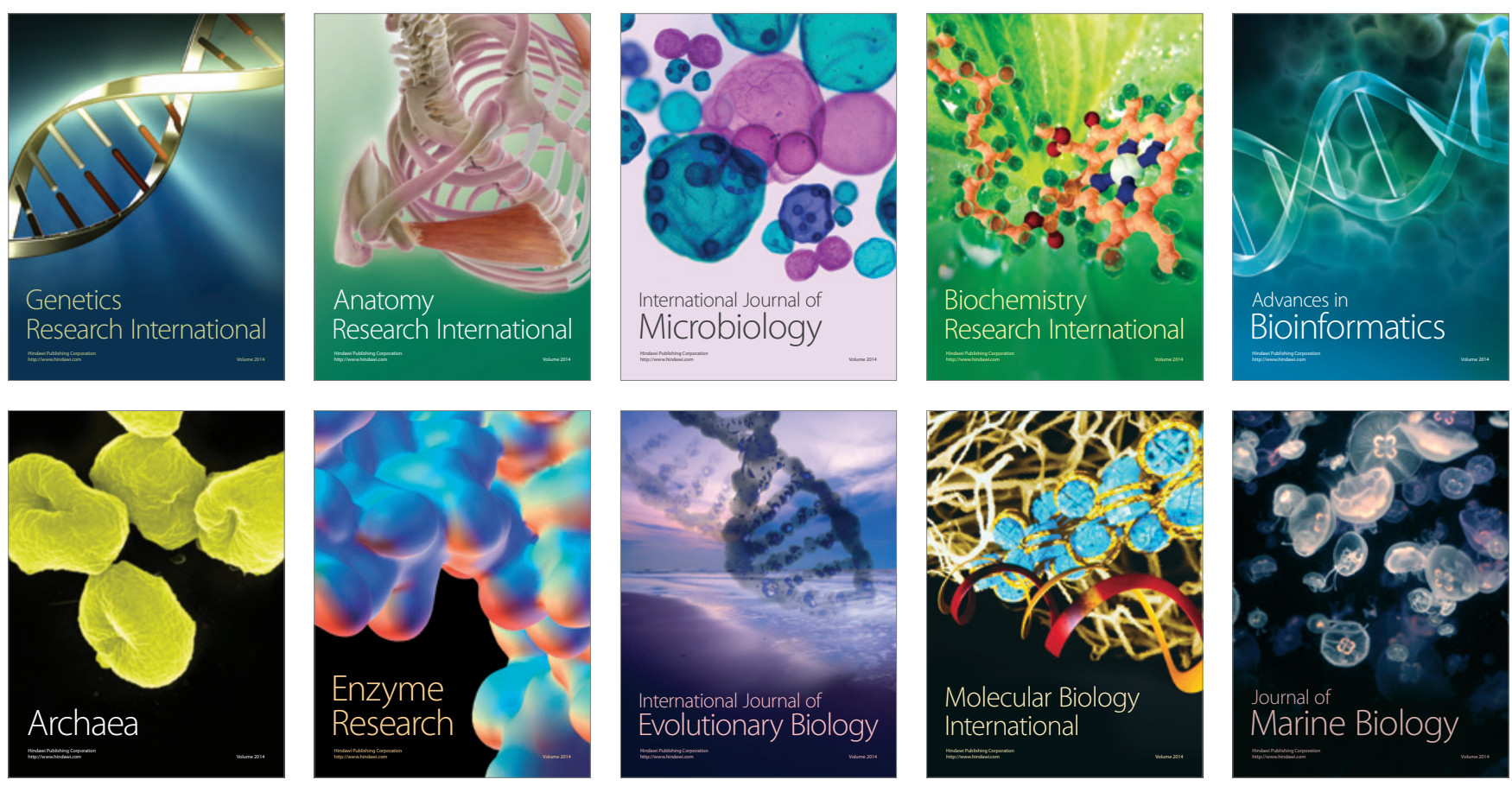